\title{
El tema del matrimonio en The Canterbury Tales de Geoffrey Chaucer
}

Claudia LUCOTTI

Universidad Nacional Autónoma de México

Siempre existen múltiples temáticas para acercarse a los textos de un autor de la talla de Geoffrey Chaucer. Este trabajo se concentrará en un tema que a mi entender, con cada lectura que se hace de su obra, adquiere mayor relevancia: el de la pareja, sobre todo en relación con el matrimonio. Una gran cantidad de sus creaciones literarias tratan estas cuestiones o aspectos relacionados con ellas, para ya en The Canterbury Tales trabajar el asunto de manera notablemente más compleja y directa.

Chaucer dedica gran parte de su obra a explorar las relaciones de pareja. Como dice Derek Brewer (1998: 57), este autor escribió sobre los sentimientos amorosos que surgen entre un hombre y una mujer con mayor profundidad que cualquiera de sus antecesores ingleses, y siempre prestando particular atención a las reacciones femeninas. Y no olvidemos que C. S. Lewis (1958: 197) ya había dicho que Chaucer fue de los poetas que contribuyeron a profundizar y refinar el concepto del amor, de modo significativo, dentro de la tradición literaria de Occidente. Si bien comparto la opinión de Brewer, creo importante subrayar que según mi lectura, The Canterbury Tales, debido a su estructura, nos presenta un panorama tan variado y tan complejo del amor y del matrimonio que nos invita a repensar en detalle el sentir de esa época con respecto al asunto, además de que nos obliga a adentrarnos en la ideología de la baja edad media relacionada con el tema para comprender de manera más completa la riqueza de sus planteamientos.

Ya desde sus obras más tempranas, e incluyendo su traducción al inglés de los primeros dos mil versos de la célebre alegoría medieval sobre el amor, Le Roman de la Rose, Chaucer demuestra un interés notable por toda esta cuestión. Sus primeros poemas se ligan de modo innegable con los diversos modos en que se relacionan hombres y mujeres. Prueba de ello son The Book of the Duchess (ca. 1369), escrito a raíz de la muerte de la duquesa Blanche, esposa del poderoso John of Gaunt, The House of Fame (ca. 1370), donde el narrador describe un largo viaje onírico que realiza para aprender 
más sobre el amor, y The Parliament of Fowls (ca.1380), que trata del amor en relación con cuestiones de índole social. Pero sin duda son los dos poemas siguientes, el famoso romance Troilus and Criseyde (ca. 1350) y The Legend of Good Women (ca. 1386), en donde Chaucer, un poeta más maduro, trabaja de manera profunda estos asuntos.

En Troilus and Criseyde explora la historia de estos infelices amantes a partir de la obligada tradición de amor cortés que su época había impuesto al tratamiento literario del tema. Sin embargo, resulta de interés apuntar aquí cómo este autor busca trazar el proceso de enamoramiento en dos tipos de personalidades radicalmente distintas. Para todo ello se interna, de maneras más trabajadas que sus antecesores, en las psicologías particulares de los dos enamorados y cómo éstos interactúan con sus contextos sociales, con consecuencias poco felices.

The Legend of Good Women, escrito en parte para compensar la visión tan problemática que presenta de Criseyde, nos muestra a un autor interesado cada vez más en el tema específico que aquí nos concierne: el de la mujer con relación al matrimonio. Habla de varias heroínas clásicas que padecieron - a pesar de su bondad y constancia - múltiples sufrimientos y abandonos a causa de sus maridos. Sin embargo, este poema, al concentrarse en una única perspectiva, resulta demasiado homogéneo y por ende poco estimulante para el lector.

Mucho de esto cambia en The Canterbury Tales (ca. 1387), en donde Chaucer explora el tema del amor y de la institución del matrimonio, sobre todo en lo que atañe a la mujer, desde una serie de perspectivas muy diversas, lo cual brinda un panorama más complejo del tema.

Para numerosos críticos, la presencia de un auténtico debate medieval sobre el matrimonio en The Canterbury Tales es un hecho, si bien no parece haber un acuerdo acerca de qué textos conforman dicho debate. Sin embargo, opino que el mayor interés que encierran estos trabajos críticos radica no tanto en el material que consideran conforma el debate sino en las relaciones que establecen entre los distintos cuentos. Para Kittredge (1960: 130159), por ejemplo, hay una clara progresión en cuanto a las distintas maneras en las que se puede vivir una relación matrimonial, y "The Franklin's Tale" sería para él el ejemplo más perfecto y acabado que encuentra Chaucer.

Este trabajo también intenta explorar las relaciones que se establecen entre los distintos cuentos que tratan el tema de la mujer y el matrimonio, pero centrándome más bien en dos aspectos que él no considera prioritarios: en primer lugar, la multiplicidad de voces y perspectivas tan característica de este autor, y en segundo lugar, el peso que tiene el contex to social e ideológico. 
El primer aspecto se relaciona con el hecho de que considero que los planteamientos de Kittredge, en lugar de hacer una lectura más completa del tema del matrimonio en The Canterbury Tales, se limitan a una única perspectiva que privilegia un determinado modelo por encima de los demás, con lo cual se pasa por alto toda la riqueza de contrastes, así como la falta explícita de conclusiones claras, todo ello tan típico de las estrategias textuales de Chaucer según la crítica más reciente.

La presencia de esta nueva vertiente de escritos influye en cómo nos acercamos a Chaucer en la actualidad. Por lo general, encontramos que su diversidad de puntos de vista — producto de un estilo muy particular- ligados a las múltiples maneras en que distintos sujetos viven o registran una experiencia, queda plasmada, de modos muy actuales, en The Canterbury Tales.

Como ejemplo de este tipo de lectura crítica podemos citar a Derek Pearsall (1979: xiv), quien analiza este rasgo de la obra de Chaucer de modo claro y convincente, para concluir que:

The encouragement that Chaucer gives to new kinds of imaginative and intellectual activity, the shock to habituated perceptions, is something that he is helped to achieve through the freedom granted to individual tales, and the constant shifting of points of view. The awareness that he stimulates, of ourselves and of the way in which we manipulate our experience of reality, is central to his concerns as a poet, and the character of individual tales and the organization of the tales as a whole are constant in their determination not to press for or permit a systematic kind of moral or ideological interpretation that will obscure or gloss over the complex realities of reading, evaluation and self-evaluation.

Esta diversidad de maneras en las que se tratan los temas, es decir la polifonía - para usar un término bajtiniano muy usado por la crítica chauceriana- de voces que emerge de la obra, es, también, un rasgo central de lo que se ha llegado a definir como la presencia de lo gótico en Chaucer, es decir, de su capacidad para crear mediante una suma de elementos disímiles.

Aquí, por cierto, quiero agregar que en relación con esta polifonía de voces, la obra de Chaucer trabaja de modo muy particular y efectivo, ya que es una característica inherente de su forma de escribir el crear textos que, por funcionar de modo más bien dramático que narrativo, permiten que cada personaje o situación mantenga una identidad propia que nosotros como lectores debemos desentrañar, en lugar de presentarlos rígidamente instalados dentro de un esquema único. Y en íntima relación con lo anterior, quiero subrayar también el peso y el efecto que tiene esta característica del esti- 
lo, incluso de la poética de Chaucer, por considerar que es justamente esta diversidad de voces que interactúan de manera permanente entre sí, pero sin perder una cierta autonomía e independencia, lo que nos impide muchas veces llegar a conclusiones fáciles o rápidas acerca de un tema, situación o personaje, lo cual a su vez le imprime un sello dialógico a su obra y la protege de caer en una simple suma de casos aislados, como sucede con muchos otros textos medievales (Benson 1986: 93).

Según mi lectura, lo que tenemos aquí es una red de personajes y situaciones que se relacionan y dialogan entre sí de forma muy abierta a lo largo de toda la obra, dentro de y entre prólogos y cuentos, para finalmente desembocar en un abigarrado tapiz, tejido con esmero, en el que figura todo, o casi todo lo que una inquieta e incluyente sensibilidad medieval podía llegar a pensar e imaginar en torno al matrimonio.

Insisto aquí en el hecho de que Chaucer es un escritor medieval, un hombre que vive en la Inglaterra del siglo XIV, y que su obra sin duda está marcada por las ideas de su tiempo. Hablar de que Chaucer es moderno, de que es nuestro contemporáneo, restándole importancia al peso de su propio contexto en su labor creativa, no conduce a nada. Sin embargo, esto no se contrapone al hecho de que su obra, como ya mencioné, posee ciertas características, que si bien en su momento eran poco comunes, actualmente se hallan próximas a mucho de lo que privilegia la literatura y la crítica.

Entonces, el que gran parte de su obra gire en torno al amor y al matrimonio - prestando siempre particular atención al papel que le tocaba desempeñar a las mujeres-, no ha de extrañarnos debido a que en su tiempo las discusiones acerca del tema de la pareja se hallaban en un momento climático.

A lo largo de toda la Edad Media, los criterios básicos en relación con el matrimonio sufren una serie de cambios notables, en la medida en que la descendencia vertical y las normas sobre la exogamia impuestas por la Iglesia eran cada vez más aceptadas por la sociedad. Esto a su vez resultó en que la figura de la mujer como esposa comenzara a cobrar una importancia creciente (Otis-Cour 2000: 171).

Sin embargo, no hay que olvidar el hecho de que estos criterios pasan por una serie de etapas distintas - lo cual no significa de modo alguno una evolución clara y positiva-, ya que muchas veces resultan conflictivos y contradictorios, e incluyen marcados retrocesos en cuanto a cómo concebían estos temas muchas de las mentes más ilustradas y escuchadas de la época.

Los escritos de los padres de la Iglesia habían dado origen a una larga y muy influyente corriente teológica que asocia a la mujer con el cuerpo, el pecado y la muerte, y busca más bien controlarla mediante una vida de en- 
cierro, silencio y castidad. Sin duda, es una visión de este tipo la que alimenta, al menos en parte, la reforma gregoriana de los siglos XI y XII, la cual busca un retorno a la pureza evangélica, a través de una cruzada que pone un énfasis muy especial en el celibato de los sacerdotes seculares, y también cuando fuera posible en el de los laicos. Para ello, esta idea de la mujer marcada por una carnalidad desbocada y, por ende, la verdadera responsable de toda falta cometida por los varones resultaba muy conveniente.

De modo paralelo, y en parte como resultado de esta creciente sensibilidad moral, sumado a toda otra serie de cuestiones de índole política y social, se profundiza el debate sobre el matrimonio, al cual se ve como la mejor solución para aquellos que no logran ejercer la continencia. Como resultado de todo esto, la relativa autonomía del matrimonio en relación con el poder de la Iglesia pronto sufrió cambios, al buscar y lograr esta institución convertirse en la autoridad dominante y utilizar su peso para reglamentar todos los aspectos relacionados con el tema. Para comienzos del siglo XII la Iglesia define al matrimonio como un sacramento indisoluble en donde lo que cuenta cada vez más es la relación íntima, basada en el consentimiento mutuo, que une a dos personas. A continuación, discute y promueve una moral y un modelo de conyugalidad ideal, que sin embargo se encontrarán escindidos debido a los varios modos en que se aborda la cuestión, sobre todo en lo que atañe al ejercicio de la sexualidad. Por un lado están las necesidades sexuales y las cuestiones hereditarias de una población civil mermada por la peste, sumado esto a una tradición clásica así como a la influencia de los preceptos principales del amor cortés que veían al sexo y la sensualidad como fuentes de placer importantes para el ser humano; por el otro, la visión negativa de la mujer y del ejercicio de la sexualidad por parte de diversos sectores de la Iglesia, aunque no de todos.

Para la Iglesia de esa época, el tema del acto sexual y el placer que encierra es de enorme trascendencia. En principio, la realización de dicho acto dentro del matrimonio sólo era aceptable si tenía fines reproductivos, aunque también se lo consideraba como una solución al problema de la lujuria y la fornicación. Sin embargo, a pesar de que es evidente que la Iglesia misma esperaba que el matrimonio se consumara, esto no resolvía el problema de la realización del acto en sí y del potencial placer que de allí podría derivarse.

Sin embargo, los pronunciamientos que hace la Iglesia por lo general se preocupan más por la conducta de los varones, y relegan a un segundo plano a la mujer. Aquí resulta de gran interés ver cómo a pesar de que en principio es sin duda el cuerpo femenino el elemento que rompe la relación armoniosa entre el hombre y Dios, no parece existir ningún planteo concre- 
to y propositivo para las mujeres en cuanto a cómo relacionarse con su cuerpo. Todo queda a nivel de represión implícita o explícita. Son muy escasos los autores que tratan el tema desde una perspectiva más concreta. No obstante ello, existen unas pocas personalidades de la época que se involucran de manera seria con el tema del placer de las mujeres, como por ejemplo Hildegarda de Bingen (1179) y Alberto Magno (1280), lo cual si bien resulta alentador, también apunta hacia lo problemático de la cuestión que no acaba por definirse en ninguna dirección concreta. En torno a esto mismo, Claude Thomasset (1992: 84) se pregunta acerca del peso que tienen en su época estas voces que toman en cuenta a la mujer para concluir que, gradualmente y con muchos tropiezos, surge una conciencia de la sexualidad femenina.

Las conclusiones optimistas a las que llega Claude Thomasset han sido cuestionadas. Sin embargo, lo que resulta indudable es la creciente importancia, si bien plena de interrogantes, que va cobrando la mujer casada a partir de su cuerpo, pero ya no sólo limitada a él, en las discusiones que se dan en torno a la familia y a los papeles que desempeñarán ahí dentro las mujeres como esposas o madres, en los últimos siglos de la Edad Media, siglos en los que éstas adquieren una creciente importancia en muchos ámbitos distintos. Un ejemplo de esto es la prolífica literatura dirigida a la mujer casada o a la joven casadera sobre el tema, en la cual se subraya la existencia de esposas ejemplares siempre sometidas a sus esposos. Asimismo, se escriben múltiples manuales y libros llenos de consejos prácticos para instruir a la mujer en el arte de gobernar una casa.

Resulta indiscutible que muchos de estos textos refuerzan y transmiten de modo creciente un modelo de pareja más y más alejado del eros carnal e incluso del eros platónico característico del amor cortés, para funcionar a partir de un sentimiento más equitativo, el affectio maritalis, en donde está presente una serie de elementos tomados del viejo concepto clásico de amistad - santo Tomás de Aquino habla del matrimonio como la amistad más grande- lo cual convierte a la pareja en una especie de sociedad conyugal. Un buen número de cartas, diarios y textos autobiográficos de fines de la Edad Media nos muestran a todo tipo de esposos y esposas funcionando juntos de manera armoniosa.

Sin embargo, esta armonía no es siempre del todo clara ya que por lo general el marido sigue ocupando una posición central, estableciéndose así una relación desigual. Pero también es cierto que toda esta literatura, y la ideología que la sustenta, al darles a las mujeres mayor presencia y responsabilidad y al prepararlas para ello, también las visualiza y las posiciona de manera nueva, lo cual tuvo consecuencias de peso, sobre todo dentro de la 
burguesía, donde debido a la creciente importancia económica de las mujeres se exacerban, por cuestiones económicas y legales, los conflictos entre los sexos. De hecho, según críticos como Wade (1986: 35), en esta época, debido al resurgimiento de una misoginia feroz, comienza la "guerra de los pantalones", la cual va acompañada de toda una literatura acorde con este sentimiento.

Se piensa que la presencia de mujeres en movimientos heréticos o semiheréticos como los cátaros o las beguinas, se debe en gran medida a querer escapar de esta creciente misoginia, pero que la reacción más importante en contra de esto se da en una nueva literatura a favor de las mujeres, muchas veces escrita incluso por ellas. Pensemos aquí, a modo de ejemplo, en la Querelle des Femmes, el famoso debate que tiene lugar en la Francia de comienzos del siglo $X V$ a raíz de la visión tan negativa de la mujer que presenta la segunda parte de Le Roman de la Rose. La Querelle resulta de gran interés sobre todo porque en este debate ya no sólo participan voces masculinas en la defensa de la mujer, sino porque también, casi por vez primera, cobra presencia la mujer que narra sus propias experiencias con su propia voz (Power 1975: 30). Sin embargo, no debemos olvidar el contexto más amplio en el cual se inserta la Querelle, un contexto en el que las actitudes misóginas se han vuelto a exacerbar, las actitudes respecto al cuerpo y la sexualidad femenina siguen siendo muy ambivalentes, y las ideas en torno al papel que le toca desempeñar a la mujer, sobre todo dentro del matrimonio, están en plena transformación.

Existen pruebas de que Chaucer estaba muy al tanto de la existencia y del peso de muchas de estas cuestiones, con sus respectivos interrogantes y debates. ${ }^{1}$ Sin duda la prueba más contundente de esto es The Canterbury Tales. En esta obra están presentes todas, o casi todas, las modalidades en las que se podían presentar dichas cuestiones, según la mentalidad de la baja edad media, y éstas siempre vistas de múltiples modos que se retroalimentan de las más variadas maneras, lo cual sí resulta un experimento muy novedoso para su tiempo.

Dentro de este espectro podemos mencionar, en primer lugar - aunque sin olvidar el hecho de estar comentando una obra que no funciona sólo de manera lineal, sino que es un tejido en donde las hebras se juntan en más de una instancia - a toda una vertiente de cuentos como "The Miller's Tale", "The Merchant's Tale" y "The Reeve's Tale”, que no sólo giran en torno a,

${ }^{1}$ Esto se ve de manera clara en "The Parson's Tale", donde Chaucer hace referencia al Postquam, un texto medieval clave, donde se recoge gran parte de los debates de la época en torno a las relaciones sexuales. 
sino que problematizan, el tema del deseo físico en su versión más descarnada, es decir, el famoso eros material que tanto preocupaba a la Iglesia medieval en contraposición a las relaciones basadas en el dilectio, es decir, un afecto más racional y manejable. En síntesis, una serie de historias que contienen la típica materia de la cual están hechos los fabliaux.

Pero, a pesar de todo ello, "The Millers Tale" tampoco se limita exclusivamente al sentir de los fabliaux tradicionales con su visión tan desagradable de las mujeres, pues aquí nos encontramos con el deseo y el placer físico vistos, en gran medida, a partir de Alison, la adúltera esposa del carpintero, la cual una y otra vez es descrita de manera gozosa mediante una serie de términos que remiten al reino animal, donde los instintos eran considerados perfectamente aceptables. Considero que este aspecto del cuento, que por cierto ocupa el segundo lugar en la colección, introduce un elemento central que influirá en toda nuestra lectura de la obras, pues nos invita - al menos en parte- a ver el ejercicio de la sexualidad, aun cuando no siga las reglas del amor cortés, como algo gozoso y placentero, sobre todo si se ve desde una perspectiva desligada de la moral cristiana.

Sin embargo, todo este goce, y hasta cierto punto inocencia, desaparece si giramos la lente para retomar estos puntos desde otras perspectivas, lo cual enriquece el diálogo que se establece entre los textos. Ejemplo de esto es "The Reeve's Tale", en donde se exploran los aspectos más crudos y desagradables que también se pueden dar en las relaciones humanas, cuando la mujer no pasa de ser tratada como una vagina.

Otra historia que gira en torno al deseo físico y al adulterio es "The Merchant's Tale", donde al clásico tema del marido viejo, es decir, el proverbial senex amans, que escoge casarse para no arder, se suma el del marido excesivamente prendado de su mujer, una característica que se manifiesta de manera tan evidente en el personaje de January que llega a bordear en la obsesión malsana. Esto a su vez permite, si bien sólo a destellos, repensar el tema del deseo físico dentro del matrimonio, y entender de modos crecientemente más complejos lo difícil de llegar a conclusiones tajantes al respecto, pues el goce y la repulsión a nivel físico no siempre se experimentan ahí donde lo indica la ley.

Esta multiplicidad de maneras de vivir, incluso de representar, una relación entre un hombre y una mujer en su aspecto más elemental, es decir, aquel relacionado con el deseo físico, no sólo se mantiene sino que se potencia cuando pasamos al trato que recibe todo ello en los romances. "The Knight's Tale" - un ejemplo perfecto del eros de corte platónico que marca el amor que sienten los muy nobles guerreros Palamon y Arcite por la bellísima y pasiva Emilia, la dama ideal, y las arduas aventuras que deben em- 
prender para poder conquistarla- nos muestra al amor cortés en su forma más pura. Sin embargo, también es cierto que el hecho de que muchas de las principales características de este cuento se repitan en "The Miller's Tale", al cual antecede, establece todo tipo de resonancias que circulan en ambas direcciones - algunas bastante hilarantes-y obliga a pensar en qué medida, por debajo de muchas diferencias formales, las relaciones entre hombres y mujeres se establecen siempre a partir de ciertos elementos comunes básicos.

A pesar de estas particularidades, éste es el romance que menos se aparta de lo tradicional y conviene más bien buscar en cuentos como "The Franklin's Tale" elementos que rompan de modo más atrevido con lo esperado, y que ejemplifiquen de manera clara esta característica de Chaucer que Brewer (1998: 85) ha definido como "a touch of extremism, of forcing things to the limit", para así volver cada vez más plural y complejo el tratamiento del tema que nos ocupa. En esta historia, que se desarrolla en el idílico mundo artúrico de los romances medievales, el tradicional triángulo amoroso damamarido-caballero aparece incluso aún más borrado ya que, si bien se mantiene en principio, una lectura más cuidadosa permite ver que todos los aspectos y las funciones tradicionales han sido desfigurados. La historia inicia donde termina la del caballero, es decir, inicia con la vida casada de la pareja, una pareja en donde muchas de las características del amor cortés se combinan ya con las de la relación matrimonial. Pero aquí lo más novedoso es la forma en la que los estereotipados papeles femeninos y masculinos, dentro de este extraño mundo de características oníricas que indican claramente que no debemos realizar una lectura por completo literal, están alterados ya que la que debe pasar por la gran prueba es la esposa. Es decir, se introduce una versión de pareja en la que, al menos parcialmente, la mujer -ya no puro cuerpo- desempeña un papel de creciente responsabilidad ligado a una compleja problemática ética. Por otra parte, el cuento incluye también un retrato interesante, más allá de lo aparentemente problemático que puede resultar, de Arveragus, al cual le toca cumplir, a medida que la historia avanza, con el papel que buscaba asignarle la Iglesia y la sociedad a un buen esposo, es decir, el de un guía racional que aconseja y educa a una esposa inexperta.

Dentro del concierto de voces polifónicas que hallamos en The Canterbury Tales, nos encontramos con otra serie de esposas que complejizan el tema, ya que en este caso son cuentos que giran en torno a mujeres y matrimonios aún más problemáticos. Tenemos, por ejemplo, a Constance en "The Man of Law's Tale", una historia que incorpora de modo claro la opinión muy difundida a finales de la Edad Media de que el amor y la sexualidad implican sufrimiento, y a la infeliz y plebeya Griselda, ese vacío humano, en 
“The Clerk's Tale", cuya abnegación y obediencia rayan incluso en lo enfermizo e invitan de modo enfático a pensar en lo que implicaría para una mujer cumplir de modo total con las exigencias de la época en cuanto a silencio, sumisión y pasividad. El mismo estudiante, así como el narrador, manifiestan de modo explícito la necesidad de repensar todo el asunto.

Sin embargo, dentro del grupo de las mujeres casadas, la voz de más peso y con mayores implicaciones para las discusiones de la época en torno a la pareja y el matrimonio, es la de Alison, la comadre de Bath. Ésta no sólo irrumpe en el texto con el poder y el caudal de una ensordecedora catarata, sino que relativiza lo negativo del monstruoso silencio que envuelve a Griselda.

Debido a la cantidad y diversidad de aspectos que toca Alison en su larguísimo prólogo, sumado a cómo se relaciona éste con su polémico cuento, el personaje no resulta fácil de resumir o clasificar, salvo en lo que concierne al derecho que se autoconcede de hablar en público, con voz y perspectiva propias, acerca de su experiencia concreta de la vida a partir del hecho de ser mujer, lo cual a su vez está íntimamente ligado con su cuerpo; todo ello desemboca en una visión nueva de la pareja, la sexualidad y el matrimonio, muy distinta de la que emerge de los discursos masculinos.

Esto se cristaliza de modo más que claro en su prólogo, en donde explora la cuestión de la posicionalidad en relación con la experiencia amorosa de manera memorable cuando dramatiza para los demás peregrinos sus conversaciones, o mejor dicho discusiones, con sus cinco difuntos maridos, y en donde se retoma el tema del deseo, la fidelidad, la obediencia y la búsqueda de igualdad. Y si bien gracias a esta dramatización le da cabida, al menos parcialmente, a las palabras y a los puntos de vista de las dos partes en conflicto, también es cierto que saca a relucir, de modo más que efectivo, la exacerbada misoginia que subyace la mayoría de los planteamientos de la época en torno a la mujer, y todo ello dentro del marco del más reciente universo del matrimonio burgués. Asimismo, el tema del ejercicio de la sexualidad por parte de la mujer dentro de distintos matrimonios y con distintos fines, que van desde el cumplir con la deuda conyugal a cambio del bienestar económico, a usar el cuerpo para gozar intensamente a nivel físico, sin jamás pensar en la obligación de procrear, le otorgan a este texto un interés permanente, además de que todo esto vuelve a ligarse con el tema inicial de la mujer y la sexualidad que encontramos en "The Miller's Tale".

Para concluir este trabajo quiero citar a Anthony Low, quien en su libro The Reinvention of Love (1993: 3-7) —donde explora cómo los cambios culturales por los que atraviesa una sociedad influyen y modifican sus modos de amar y las formas sociales y literarias específicas que adquieren es- 
tos modos- dice en su introducción acerca de sir Philip Sidney que "With regard to love, Sidney was not only a last brilliant exemplar of the older, aristocratic Petrarchan tradition, but an important precursor of a centurylong process of change and reinvention". Concluye esta sección —en la que hace un breve recuento de todos aquellos autores que contribuyeron a este cambio - mencionando que Spencer, Shakespeare y Jonson "played a critical role in turning love away from courtly origins toward a new ideal of mutual love and courtship leading to marriage".

Sin embargo, si bien coincido con Low en lo general, considero importante volver a hacer énfasis en que ya Chaucer - con su estilo tan particular-doscientos años antes había explorado insistentemente el tema de muy variados modos y con una complejidad creciente. Es más, el que mucha de su obra gire, de hecho, en torno a las mujeres y el matrimonio, no ha de extrañarnos en absoluto debido al interés que había cobrado el tema en su época. Por otra parte, resulta fundamental tomar todo esto en cuenta para poder apreciar de modo más completo comentarios como los de Brewer y Lewis acerca del papel protagónico que tiene Chaucer como poeta del amor.

\section{Bibliografía}

Benson, C. David. 1986. "The Canterbury Tales: Personal drama or experiments in poetic variety?" The Cambridge Chaucer Companion. Ed. de Piero Boitani y Jill Mann. Cambridge: Cambridge University Press.

Brewer, Derek. 1998. A New Introduction to Chaucer. Londres: Longman.

Kittredge, G. L. 1960. "Chaucer's Discussion of Marriage”. Chaucer Criticism. The Canterbury Tales. Ed. de Richard Schoeck y Jerome Taylor. Notre Dame/Londres: University of Notre Dame Press.

Lewis, C. S. 1977. The Allegory of Love. A Study in Medieval Tradition.2a. ed. Oxford: Oxford University Press.

Low, Anthony. 1993. The Reinvention of Love. Poetry, Politics and Culture From Sidney to Milton. Cambridge: Cambridge University Press.

Otis-Cour, Leah. 2000. Historia de la pareja en la Edad Media. Placer y amor. Madrid: Siglo XXI. 
$46 \square$ EL TEMA DEL MATRIMONIO EN THE CANTERBURY TALES

Pearsall, Derek. 1979. The Canterbury Tales. Londres: Unwin Hyman.

Power, Eileen. 1975. Medieval Women. Cambridge: Cambridge University Press.

Thomasset, Claude. 1992. "La naturaleza de la mujer". Historia de las mujeres. La Edad Media. Vol. 2. Ed. de Georges Duby y Michelle Perrot. Madrid: Taurus.

Wade Labarge, Margaret. 1986. A Small Sound of the Trumpet. Boston: Beacon Press. 